\title{
FE ANALYSIS OF A COUPLED ENERGETIC-STATISTICAL SIZE EFFECT IN PLAIN CONCRETE BEAMS WITH VARYING MATERIAL PROPERTIES
}

\author{
E.SYROKA-KOROL ${ }^{*}$ AND J.TEJCHMAN ${ }^{\dagger}$ \\ *Gdansk University of Technology \\ Gdansk, Poland \\ e-mail: ewelina.korol@pg.gda.pl \\ ${ }^{\dagger}$ Gdansk University of Technology \\ Gdansk, Poland \\ e-mail: tejchmk@.pg.gda.pl
}

Key words: Concrete beams, Cross-correlation, Random fields, Size effect

\begin{abstract}
The numerical FE investigations of a coupled energetic-statistical size effect in unnotched concrete beams of similar geometry under quasi-static three point bending were performed within elasto-plasticity with non-local softening. The stochastic FE analyses were carried out with three different beam sizes. Deterministic calculations were performed with the uniform distribution of a uniaxial tensile strength. In statistical calculations cross-correlated random fields were used to describe the spatial fluctuation of material randomness. Stochastic simulations were made with varying uniaxial tensile strength, initial fracture energy and elastic modulus. The effect of independently and simultaneously varying material parameter was investigated.
\end{abstract}

\section{INTRODUCTION}

A size effect in quasi-brittle materials such as concrete causes that both the nominal structural strength (the measure of the ultimate load expressed in stress units) and material brittleness decrease with increasing characteristic specimen size $D$. Thus, concrete becomes ductile on a small scale and perfectly brittle on a sufficiently large scale. This behavior can be explained on the one hand by energetic (deterministic) phenomena caused by strain localization of a finite volume, depending on a characteristic length of microstructure $l_{c}$ [1]. On the other hand, a statistical effect may be found in a heterogeneous nature of concrete with randomly distributed material properties [2]. The first statistical theory of size effect has been introduced by Weibull [2] and assumes that a structure is as strong as its weakest component. However, the statistical size effect by Weibull ignores a spatial correlation between local material properties and an energetic (deterministic) size effect. As a result, the pure statistical theory of size effect by Weibull provides the unrealistically low nominal strength of small and medium specimens. The most realistic energetic size effect theory has been given by Bazant [1]. Structures of Type I (e.g. a plain concrete beam without a notch) which are sensitive to material randomness are described by a coupled energetic-statistical formula (Eq.1). The nominal strength depending on $D$ is defined as a non-linear function approaching a plasticity limit for small sizes and Weibull asymptote for large sizes (Fig.1a). 


$$
\sigma_{N}(D)=f_{r}^{\infty}\left(\left(\frac{L_{o}}{D+L_{o}}\right)^{\frac{r n}{m}}+\frac{r D_{b}}{D+l_{p}}\right)^{\frac{1}{r}}
$$

where $m$ is the dimensionless Weibull modulus (shape parameter of Weibull distribution) responsible for the slope of a large-size asymptote and $n$ is the number of spatial dimensions in which the structure is scaled. Thus, the mean size effect is separately divided into a stochastic part and deterministic one. The parameter $D_{b}$ drives the transition from elastic-brittle to quasi-brittle and $L_{o}$ drives it from constant property to local Weibull via strength random field.

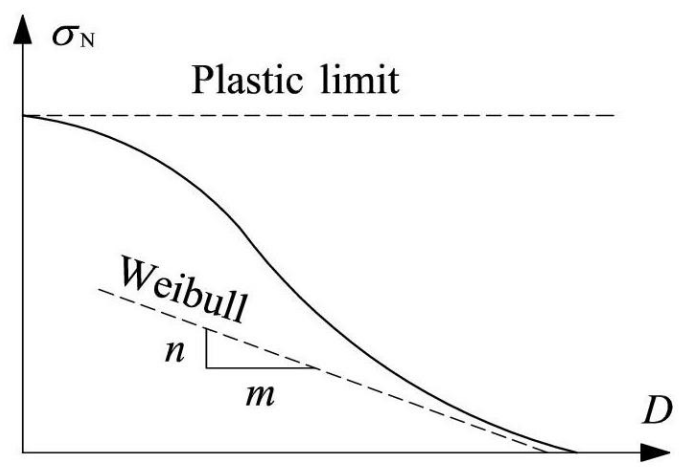

a)

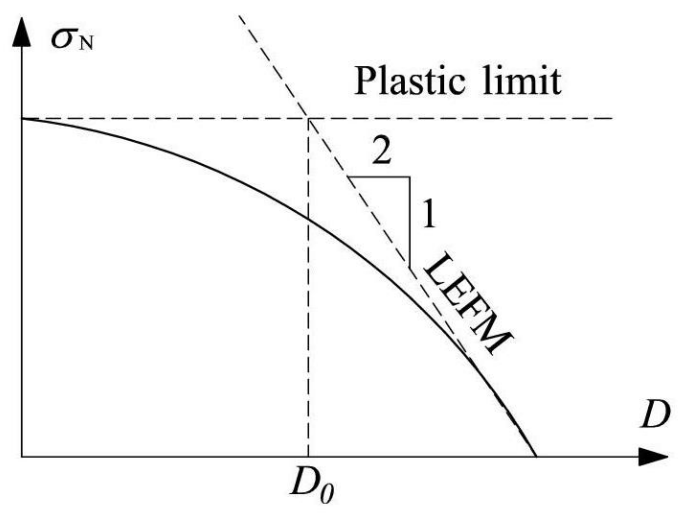

b)

Figure 1: Energetic-statistical size effect Type I (a) and energetic size effect Type II (b) by Bazant [1],[3]

Structures of Type II (e.g. notched concrete beam or reinforced concrete beam failing due to shear) are describe by Equation 2 which ignores the statistical effect i.e. the mean strength is not affected by the material randomness.

$$
\sigma_{N}(D)=\frac{B f_{t}^{\prime}}{\sqrt{1+D / D_{0}}}
$$

here $f_{t}$ ' is a uniaxial tensile strength, $B$ is a dimensionless parameter characterizing the geometry and $D_{0}$ is a transitional structure size. The nominal strength defined by SEL Type 2 is transitional between plasticity and LEFM (Fig.1b). Unknown parameters in Eqs.1-2 can be identified simply by fitting experimental or numerical results. Some of those parameters can also be found by considering ideally plastic (very small size) and ideally brittle (very large size) structures.

\section{FE-INPUT DATA}

The FE analysis was performed for plain concrete beams experimentally investigated by Hoover et.al. [4]. Geometrically similar beams scaled in two dimension i.e. depth (height) $D$ and span $L_{\text {eff }}$ were tested (Fig.2). The beam height changed from $40 \mathrm{~mm}$ up to $500 \mathrm{~mm}$ while the beam thickness $b=40 \mathrm{~mm}$ was kept constant for all sizes. Sets of beams of 18 different geometries were used. Altogether, 140 beams were tested with the size range of $1: 12.5$ and with relative notch depths $\alpha=a / D$ ( $a$ - notch depth) ranging from 0 to $30 \%$ of the beam depth. The tiny beam had $D=40 \mathrm{~mm}$ depth and four different relative notch depth $\alpha=0.3,0.15,0.075$ and 0 . The small size beam was $D=93 \mathrm{~mm}$ high with a relative notch $\alpha=0.3,0.15,0.075$ and 0 . The medium size beam had a depth $D=215 \mathrm{~mm}$ and five different relative notch depth $\alpha=0.3,0.15$ and $0.075,0.025$ and 0 . The large size beam of $D=500 \mathrm{~mm}$ possessed also five relative notch depth $\alpha=0.3,0.15$ and $0.075,0.025$ and 0 . All tests [4] were performed under opening displacement control. Basic material characteristics such as an elastic modulus $E$, flexural tensile strength $f_{r}$ and Poisson ratio $v$ were measured as required by ASTM standard. Material parameters are given in Tab.1 


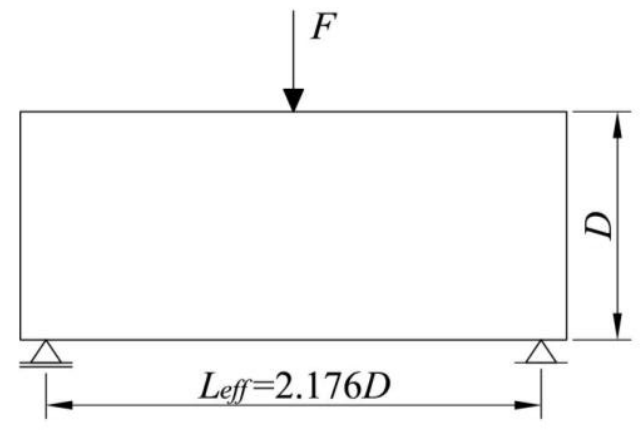

Figure 2: Geometry and boundary conditions of analyzed beams.

The two-dimensional plain stress FEanalysis of free-supported concrete beams was performed with a fine mesh in the mid-part of a beam where a localized zone was expected to develop (the area with a fine mesh was $D \times D$ ). The width of a finite element did not exceed $1.5 \mathrm{~mm}$. The quadrilateral elements divided into triangular elements were used to avoid volumetric locking [5]. For all beam sizes the width of finite element was used the same whereas the height ca. $20 \mathrm{~mm}$ changed slightly by less than $10 \%$.

Table 1: Material deterministic parameters

\begin{tabular}{cc}
\hline Parameter & Value \\
\hline$E$ & $41.24 \mathrm{GPa}$ \\
\hline$v$ & 0.17 \\
\hline$f_{t}{ }^{\prime}$ & $3.92 \mathrm{MPa}$ \\
\hline$\sigma_{k}$ & $0.588 \mathrm{MPa}$ \\
\hline$\kappa_{k}$ & $13.386 \mathrm{E}-4$ \\
\hline$\kappa_{f}$ & $62.25 \mathrm{e}-4$ \\
\hline
\end{tabular}

\section{CONCRETE MODEL}

To properly describe strain localization and to include a characteristic length of microstructure for simulations of a deterministic size effect, a non-local theory was used as a regularization technique [6]. In the calculations, the softening parameter in tension $\kappa$ was assumed to be non-local $(\bar{\kappa})$ [7]. As a weighting function, a Gauss distribution function was used. The characteristic length of micro-structure $l_{c}=5 \mathrm{~mm}$ defined the averaging radius.

To describe the behavior of concrete under tension during three-point bending, a Rankine criterion was used, for which the yield function $f$ with isotropic softening defined as

$$
f=\max \left(\sigma_{1}, \sigma_{2}, \sigma_{3}\right)-\sigma_{t}(\kappa)
$$

where $\sigma_{i}, i=1,2,3$, are the principal stresses, $\sigma_{t}$ is the tensile yield stress and $\kappa$ denotes the softening parameter equal to the maximum principal plastic strain $\varepsilon_{l p}$. The associated flow rule was assumed. To model the concrete softening under tension the bilinear function was chosen (Fig.3) as recommended by Bazant and Hoover [8]. The initial fracture energy affecting the beam strength is defined as $G_{f}=0.5 \times f_{t}{ }^{\prime} \times \kappa_{l} \times w_{l o c}\left(w_{l o c}-\right.$ the width of a localized zone, $\left.w_{l o c}=3 \times l_{c}[6]\right)$. Whereas the total fracture energy affecting mainly the softening is $G_{F}=\mathrm{G}_{f}+0.5 \times \sigma_{k} \times\left(\kappa_{k}-\kappa_{l}\right) \times w_{l o c}$. The ratio $G_{F} / G_{f}=1.42$ was found by Bazant and Hoover [7] to fit all experimental results. Material parameters used in our study [Tab.1] were kept the same for all geometries and most of them where measured experimentally by Hoover et.al. [4]. Referring to deterministic simulations all material parameters were constant.

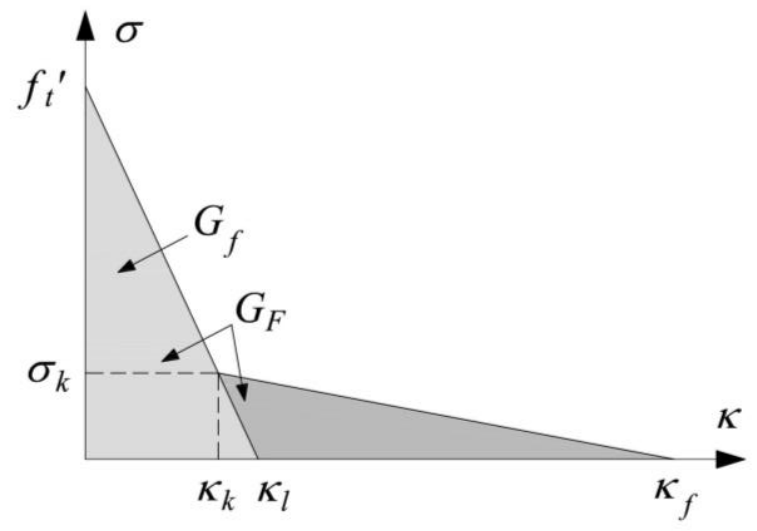

Figure 3: Bilinear softening after Bazant and Hoover (2014) [7]

\subsection{Stochastic model}

Stochastic simulations were performed assuming random, spatially correlated distribution of local material properties. Random fields were generated using Karhunen-Loëve expansion and the waveletGalerkin approach [7]. The stratified sampling 
method was used to limit the number of samples. Further FE analysis was performed for chosen set of 12 samples (i.e. 12 random fields [9]). The spatial correlation was described by a homogenous square exponential autocorrelation function with an autocorrelation length $l_{c o r}=80 \mathrm{~mm}$ :

$$
C\left(x_{1}, x_{2}\right)=\exp \left(-\frac{\tau^{2}}{l_{c o r}^{2}}\right)
$$

where $x_{1}, x_{2}$ - points coordinates, $\tau=\left|x_{1}-x_{2}\right|$ is a distance between two points.

Gauss distribution function was used with the prescribed expected (mean) value $\mu$ and standard deviation $s_{d e v}$ producing $\operatorname{cov}=0.12$ (cov $=s_{d e v} / \mu$ is a material coefficient of variation). The mean value of a varying material parameter was the same as deterministic value. The symmetrical probability distribution function was adopted (instead of unsymmetrical Weibull) because specimens had small sizes.

Material parameters assumed to be random were: uniaxial tensile strength $f_{t}$, initial fracture energy $G_{f}$ (the ratio $G_{F} / G_{f}=1.42$ was kept constant) and elastic modulus $E$. Spatial distributions of $f_{t}$ ' and $G_{f}$ were simply crosscorrelated whereas elastic modulus $E$ was calculated so as to give constant Irwin characteristic length $L_{c h}=E \times G_{f} /\left(f_{t}^{\prime}\right)^{2}$. The procedure to generate simply cross-correlated random fields was adopted from Vorechovsky (2008) [10]. A strong and positive crosscorrelation with $r=0.9$ was assumed for $f_{t}$ ' and $G_{f}$.

\section{FE RESULTS}

\subsection{Deterministic simulations}

Deterministic simulations were performed for all beam sizes and relative notch depths $\alpha$. Summary results are given in Tab.2 together with experimental findings $\left(\sigma^{F E}{ }_{N}\right.$ is a nominal strength from deterministic FE calculation and $\sigma_{N}^{T}$ is a mean nominal strength from test [4]). From Tab. 2 it might be seen the agreement between deterministic FE results and experiment is acceptable (an average error is 8\%) and generally the difference becomes stronger when the relative notch depth decreases. The reduction of calculated deterministic nominal strength with increasing beam depth $D$ is similar for relative notch length $\alpha=0.3,0.15$ and 0.075 and reaches ca $55 \%$ when $D$ increases from 40 up to $500 \mathrm{~mm}$. Whereas for beams without notches $(\alpha=0)$ calculated reduction is lower ca. $40 \%$ which demonstrates the energetic size effect is weaker.

Table 2: FE deterministic and experimental results [4]

\begin{tabular}{cccc}
\hline$D[\mathrm{~mm}]$ & $\alpha=a / D$ & $\sigma^{F E}{ }_{N}[\mathrm{MPa}]$ & $\sigma_{N}^{T}[\mathrm{MPa}]$ \\
\hline 40 & 0 & 8.26 & 7.76 \\
\hline 40 & 0.075 & 7.41 & 6.69 \\
\hline 40 & 0.15 & 6.43 & 5.38 \\
\hline 40 & 0.3 & 4.59 & 3.55 \\
\hline 93 & 0 & 6.87 & 7.35 \\
\hline 93 & 0.075 & 5.73 & 5.49 \\
\hline 93 & 0.15 & 4.84 & 4.54 \\
\hline 93 & 0.3 & 3.36 & 3.04 \\
\hline 215 & 0 & 5.67 & 6.30 \\
\hline 215 & 0.025 & 5.20 & 5.32 \\
\hline 215 & 0.075 & 4.51 & 4.59 \\
\hline 215 & 0.15 & 3.75 & 3.68 \\
\hline 215 & 0.3 & 2.57 & 2.55 \\
\hline 500 & 0 & 4.90 & 5.96 \\
\hline 500 & 0.025 & 4.23 & 4.71 \\
\hline 500 & 0.075 & 3.50 & 3.63 \\
\hline 500 & 0.15 & 2.83 & 2.93 \\
\hline 500 & 0.3 & 1.94 & 1.88 \\
\hline
\end{tabular}

Figure 4 presents calculated loaddisplacement (crack mouth opening displacement) curves for three different sizes of unnotched beams (small $D=93 \mathrm{~mm}$, medium $D=215 \mathrm{~mm}$ and large $D=500 \mathrm{~mm}$ size beam) which were further used in stochastic analysis. Calculated curves are compared to experimental measurements (the lowest, average and highest result registered experimentally). In all cases the simulated failure force and softening converge to experimental findings. The initial part of experimental curves up to the peak load indicates the elastic modulus might be different in particular specimens. The calculated ultimate load is always between the 
highest and lowest experimental peak load (however it is generally lower than the mean experimental strength).

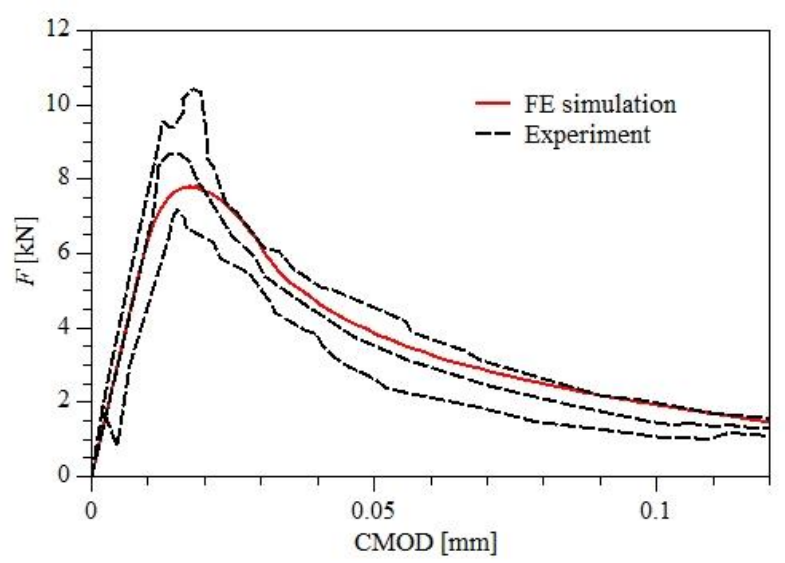

a)

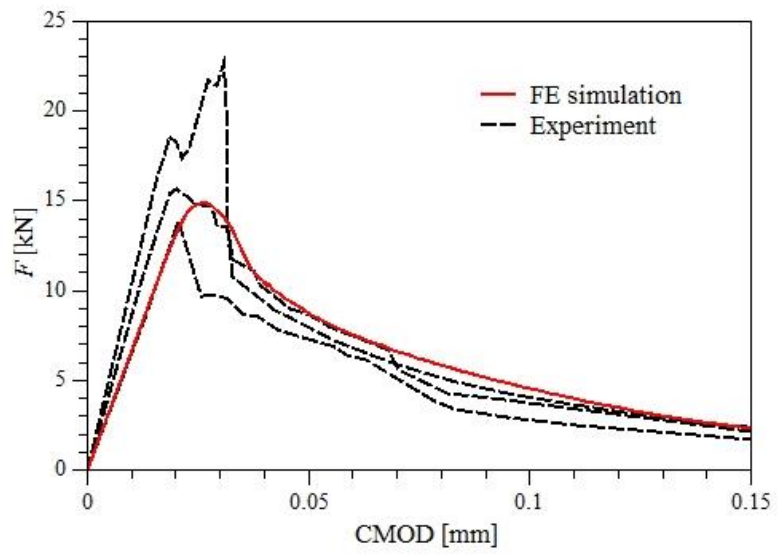

b)

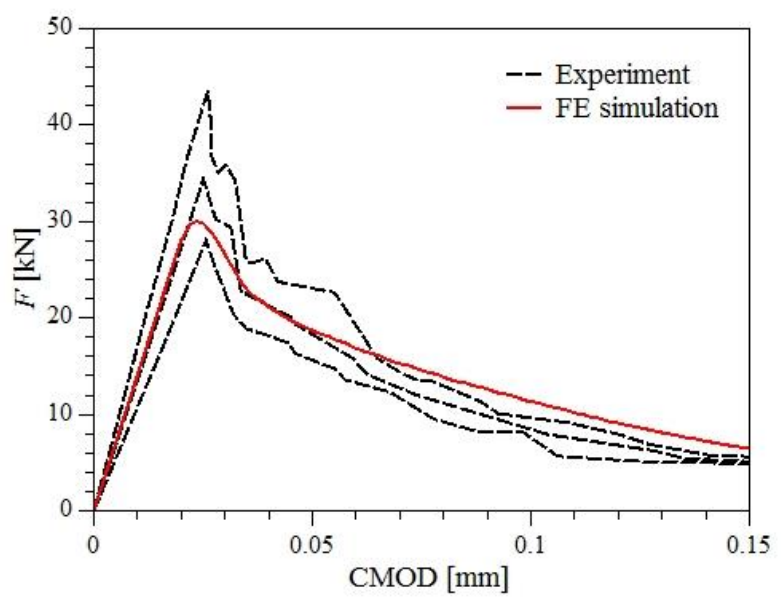

c)

Figure 4: Deterministic load-CMOD curves compared to experimental results [4] with $\alpha=0$; a) $D=93 \mathrm{~mm}$, b) $D=215 \mathrm{~mm}$, c) $D=500 \mathrm{~mm}$

\subsection{Stochastic simulations}

Three beam sizes $D=93,215$ and $500 \mathrm{~mm}$ were investigated. The tiny beam with $D=40 \mathrm{~mm}$ was ignored in statistical analysis because its size is smaller than assumed autocorrelation length $l_{\text {cor }}=80 \mathrm{~mm}$. Stochastic analysis was initially performed with only one random material parameter while keeping other parameters constant. The following three combinations were examined: a) random $f_{t}$, and constant $G_{f}$ and $E$, b) random $G_{f}$ and constant $f_{t}$ ' and $E, \mathrm{c}$ ) random $E$ and constant $f_{t}$ ' and $G_{f}$. Next, the analysis was run for all three spatially varying material parameters: $f_{t}$, $G_{f}$ and $E$ (assuming $r=0.9$ for $f_{t}$ ' and $G_{f}$ and constant $\left.L_{c h}=E \times G_{f} f\left(f_{t}^{\prime}\right)^{2}\right)$. Figure 5a compares the effect of spatially varying $E$ ( $f_{t}$ ' and $G_{f}$ are constant). It might be seen that the elastic modulus besides changing the initial slope also slightly affects the peak load (it delays or accelerates the strain localization due to changing $\varepsilon_{0}=f_{t}^{\prime} / E$ ).

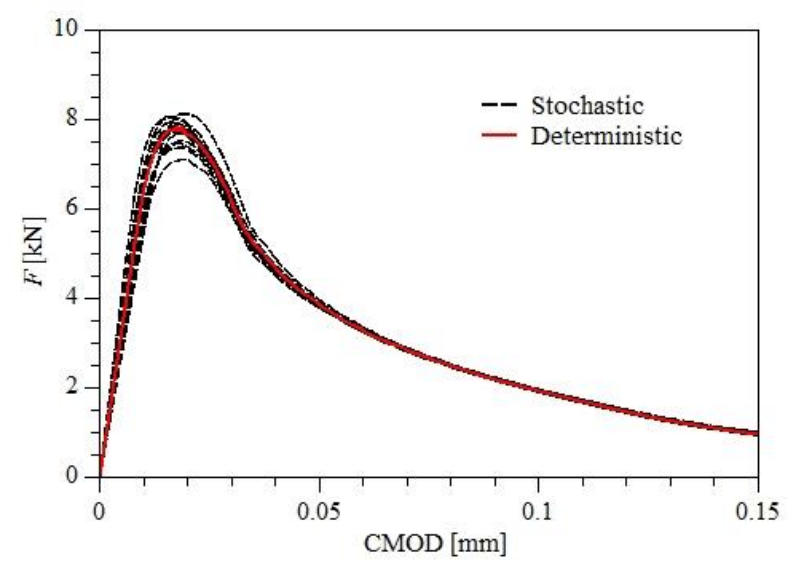

a)

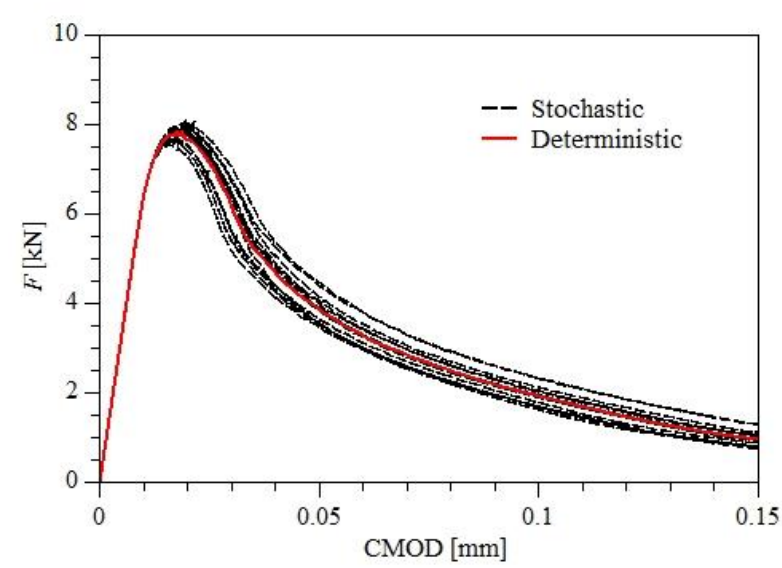

b) 


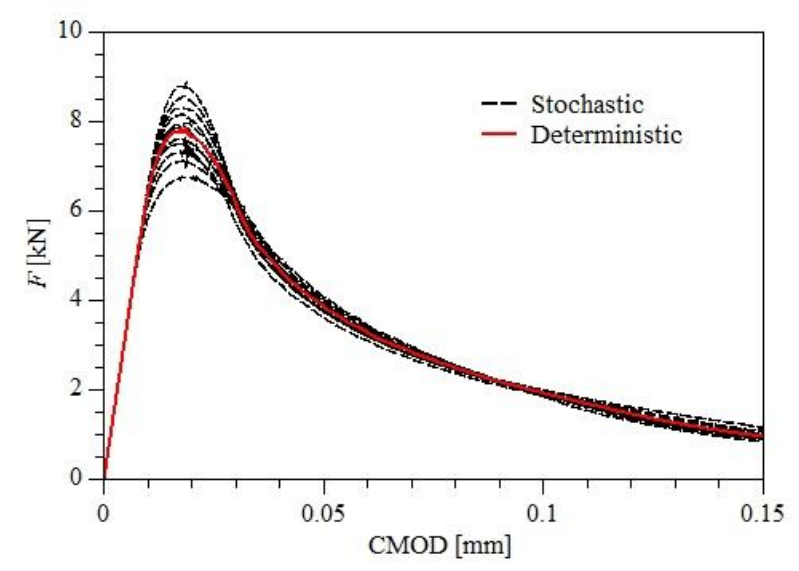

c)

Figure 5: Stochastic load-CMOD curves compared to deterministic results for small beam $D=93 \mathrm{~mm}$ with $\alpha=0$; a) random elastic modulus $E$, b) random initial fracture energy $G_{f}$, c) random tensile strength $f_{t}$.'

The mean ultimate load is $7.75 \mathrm{kN}$ and changes between $7.11 \mathrm{kN}$ and $8.13 \mathrm{kN}$. Fig. $5 \mathrm{~b}$ shows results for varying initial fracture energy $G_{f}$ together with a total fracture energy $G_{F}\left(f_{t}\right.$ ' and $E$ are constant). The mean ultimate load is quiet similar to previous case and reaches $7.82 \mathrm{kN}$. However this time the failure force varies merely from $7.55 \mathrm{kN}$ up to $8.04 \mathrm{kN}$. Hence, the fracture energy has a weaker effect on the peak load but has an effect on the structural softening. Referring to results for fluctuating tensile strength $f_{t}$ ' given in Fig. $5 c$ ( $G_{f}$ and $E$ are constant) it's clear the peak load strongly varies. The mean failure force is $7.82 \mathrm{kN}$ and changes from $6.77 \mathrm{kN}$ up to $8.82 \mathrm{kN}$. It can be concluded for the mean strength and statistical size effect the crucial parameter is the axial tensile strength $f_{t}$ '.

Figure 6 presents results for simultaneously varying $f_{t},, G_{f}$ and $E$. The calculated mean failure forces (together with a minimum and maximum) are $7.79 \mathrm{kN}(6.81 \div 9.31 \mathrm{kN})$, $14.29 \mathrm{kN}(11.81 \div 16.05 \mathrm{kN})$ and $28.24 \mathrm{kN}$ $(25.12 \div 32.34 \mathrm{kN})$ for small $(D=93 \mathrm{~mm})$, medium $(D=215 \mathrm{~mm})$ and large $(D=500 \mathrm{~mm})$ size beam respectively. The corresponding mean nominal strengths are as follows: $6.82 \mathrm{MPa} \quad(6.87 \mathrm{MPa}$ in deterministic simulation), $\quad 5.42 \mathrm{MPa} \quad(5.67 \mathrm{MPa}$ in deterministic simulation) and 4.61 $\mathrm{MPa}(4.90$ $\mathrm{MPa}$ in deterministic simulation).

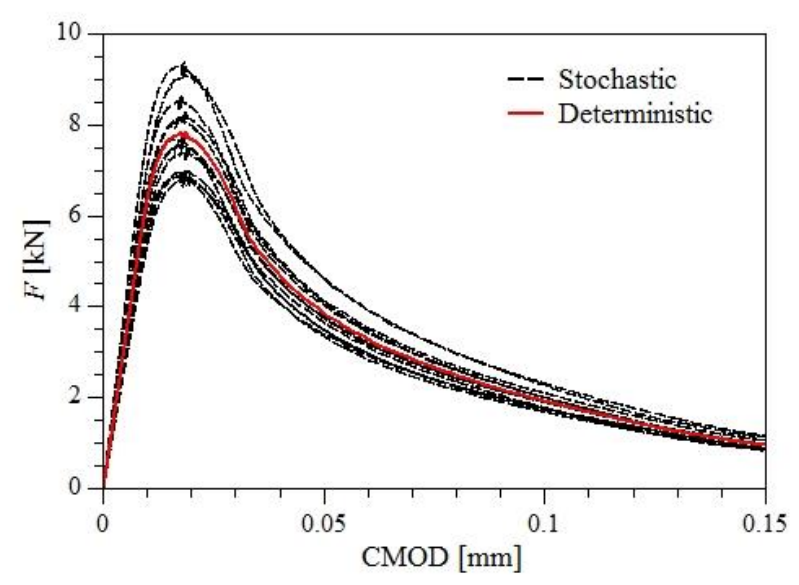

a)

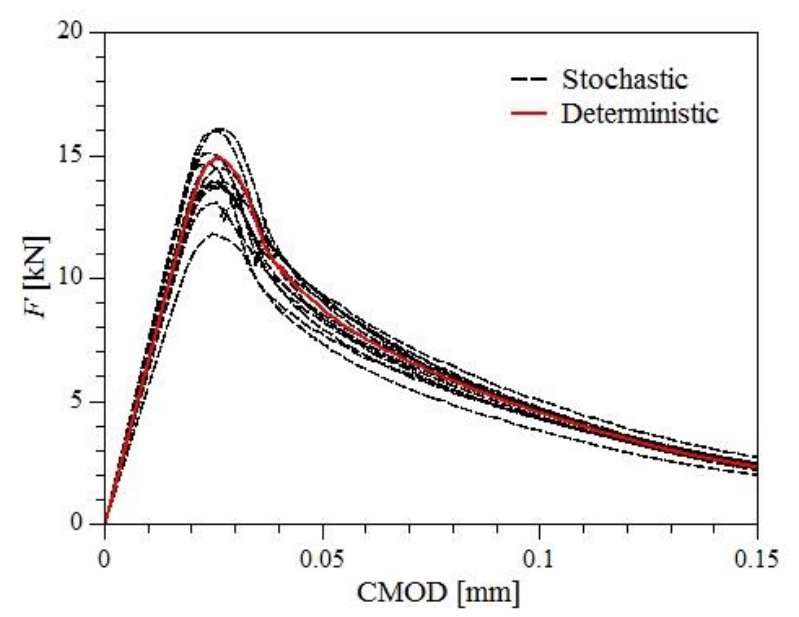

b)

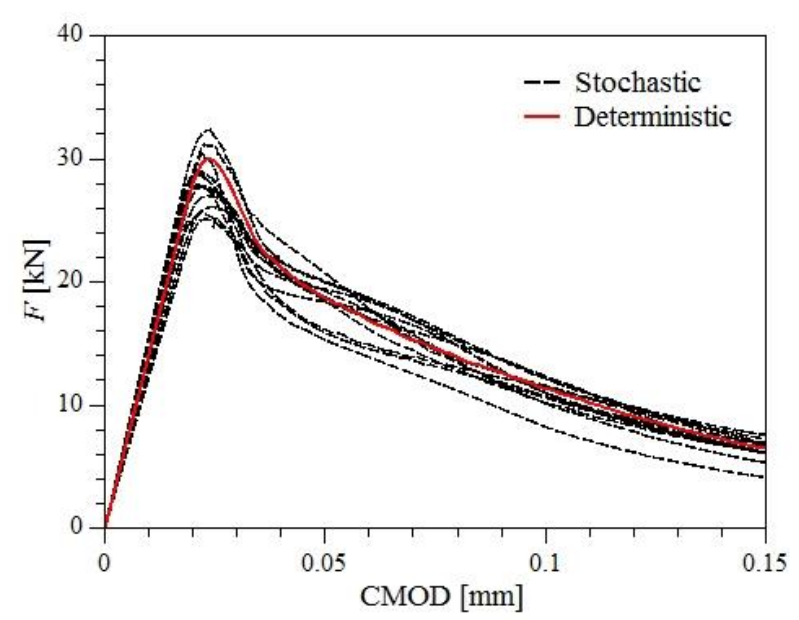

c)

Figure 6: Stochastic load-CMOD curves with simultaneously varying $f_{t}, G_{f}$ and $E$ compared to deterministic results; a) $D=93 \mathrm{~mm}$, b) $D=215 \mathrm{~mm}$, c) $D=500 \mathrm{~mm}$

The difference between mean stochastic strength and deterministic strength is very low even for the large size beam it is about $6 \%$. 
The reason of negligible statistical part of size effect is that beams are relatively short (the statistical size effect is stronger in longer beams, compare [9]). However the effect of fluctuating material parameters has a strong influence upon the scatter of the failure force and the shape of a softening.

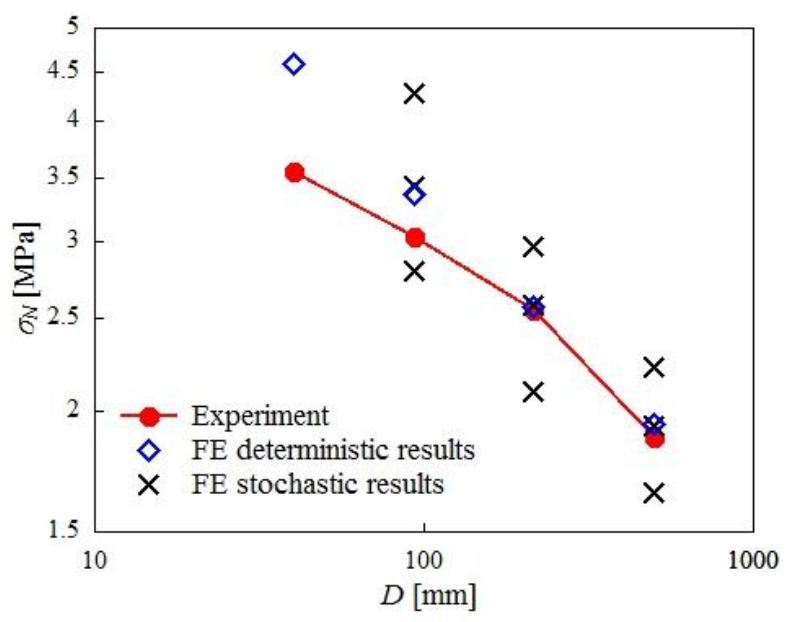

a)

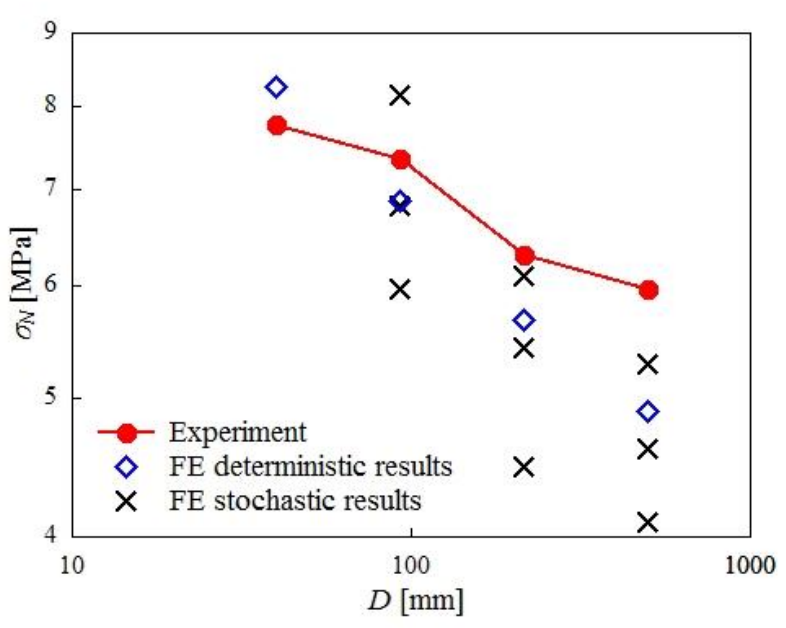

b)

Figure 7: Comparison of mean nominal strengths from experiment [4] with FE deterministic results and stochastic results (mean, maximum and minimum strength obtained for simultaneously varying $f_{t}{ }^{\prime}, G_{f}$ and

$$
E \text { ); a) } \alpha=0.3, \text { b) } \alpha=0 \text {. }
$$

Comparing numerical results in Fig.7 one might see that the best agreement with experimental mean strengths was obtain for notched concrete beams with $\alpha=0.3$. In the case of unnotched beams experiments show much weaker energetic size effect on the nominal strength. However FE deterministic results are still in the range of experimentally obtained maximum and minimum values (compare Fig.4). The problem with matching experimental results with a standard nonlocal concrete model was lately described in details by Havlásek et. al. (2016) [11].

\section{CONCLUSIONS}

The non-local elasto-plastic concrete model with a bilinear softening is able to correctly reproduce experimental results for plain concrete beams under bending. Both the peak load and the structural softening converge to the experimental findings. The nominal flexural strength of plain concrete beams strongly decreases with an increasing beam depth $D$. The reduction of a deterministic nominal strength is stronger with reference to beams with a notch i.e. structures of Type II. Material randomness in the form of crosscorrelated and spatially varying uniaxial tensile strength $f_{t}$, initial fracture energy $G_{f}$ and elastic modulus $E$ (assuming constant Irwin's characteristic length) does not significantly change the results comparing with those with singly varying $f_{t}$ '. In the case of analyzed relatively short unnotched beams the statistical part of size effect might be ignored since the nominal strength is reduced with increasing size mainly because of an energetic reasons. The material randomness strongly affects the scatter of results including the ultimate load and structural softening with reference to both notched and unnotched beams.

\section{REFERENCES}

[1] Bazant Z.P. and Planas J. 1998. Fracture and size effect in concrete and other quasi-brittle materials. CRC Press LLC.

[2] Weibull W. 1951. A statistical theory of the strength of materials. J Appl Mech, 18(9):293-7.

[3] Bazant Z.P., Vorechovsky M., Novak D. 2007. Asymptotic prediction of energetic-statistical size effect from 
deterministic finite-element solutions. J Eng Mech ASCE, 133(2):153-62.

[4] Hoover, C.G., Bazant, Z.P., Vorel, J., Wendner, R. and Hubler, M.H. 2013. Comprehensive concrete frecture tests: Description and results. Engineering Fracture Mechanincs 114:92-103.

[5] Groen A.E., 1997. Three dimensional elasto-plastic analysis of soil. PhD Thesis,Delft University, The Netherlands

[6] Bazant ZP, Jirásek M. 2002. Nonlocal integral formulations of plasticity and damage: survey of progress. J Eng. Mech, 128(11):1119-49.

[7] Syroka-Korol, E, Tejchman, J and Mróz Z. 2013. FE calculations of a deterministic and statistical size effect in concrete under bending within stochastic elasto-plasticity and non-local softening. Engineering Structures 48:205-219.

[8] Bazant, Z.P. and Hoover, C.H. 2014. Cohesive crack, size effect, crack band and work-of-fracture models compared to comprehensive concrete fracture tests. Int J Fract 187:133-143.

[9] Syroka-Korol E., Tejchman, J., Mróz Z. 2015. FE investigations of the effect of fluctuating local tensile strength on coupled energetic-statistical size effect in concrete beams, Engineering Structures, 103:239-259.

[10] Vorechovsky, M. 2008. Simulation of simply cross correlated random fields by series expansion methods. Structural Safety 30:337-363.

[11] Havlásek P., Grassl P. and Jirásek M. 2016. Prediction of size effect on strength of quasi-brittle materials based on integral-type nonlocal models. Engineering Fracture Mechanics. 157:72-85. 